\title{
Research and Application of Lightning Protection for Overhead Line of High-speed Railway
}

\author{
Yu Shudan ${ }^{1}$ \\ School of Electron and Information, Nanchang Institute of \\ Technology, \\ Nanchang 330044, China
}

\author{
Cai Peng ${ }^{3}$
}

Nanchang -Jiujiang Intercity Railway Co.,Ltd, Nanchang 330002, China

\author{
Wang Wei ${ }^{2}$ \\ School of Electrical and Automation Engineering, East \\ China Jiaotong University, \\ Nanchang 330013, China \\ Zhou Xiaojing ${ }^{4}$ \\ Department of Power Management, China Railway \\ Administration Nanchang Group Co., Ltd. \\ Nanchang 330002, China
}

\begin{abstract}
Lightning is a common discharge phenomenon in nature. Lightning is also a disaster for human society. The scope of lightning disasters covers all filed of production and life. As a space for passengers to wait, collect and distribute, the comprehensive overhead line of railways has many stayers. In order to create a comfortable and safe waiting environment for passengers, a large number of cables for communication and signals are installed in overhead lines. It increases the difficulty of electromagnetic pulses protection against lightning. Therefore, it is important to realize a normalize production of equipment standard with comprehensive prevention and overall coordination to ensure the safety of railway personnel, production operations and equipment in accordance with the priority of railway, the lightning protection standards of various equipment and buildings, and the lightning storm conditions in various places.
\end{abstract}

Keywords-High-speed railway; overhead line; lightning protection; Monte Carlo simulation

\section{TYPES AND HAZARDS OF LIGHTNING}

Generally, lightning strikes are divided into direct lightning, induction lightning and spherical lightning. Direct lightning is that thunder discharges directly to the earth. Inductive lightning is the secondary discharge of high-voltage and low-voltage areas where the resistance of the affected area is large after direct lightning. Spherical lightning is rare. But the spherical lightning will enter into the building through the electronic circuit and even damage the personal life safety.

Indirect hazards of lightning strikes include electrostatic induction over-voltage effect, electromagnetic pulse overvoltage effect, and electromagnetic induction magnetic field strength effect. The electrostatic induction over-voltage is that the high voltage area where the resistance of the affected area is large is easy to occur partial spark discharge or secondary lightning strike to low voltage area. The electromagnetic pulse over-voltage is that electronic equipment interrupted or damaged by direct radiation electrical measurement of the pulsed electric field generated by the lightning strike current.
The electromagnetic induction magnetic field strength effect is that the large electromotive force of electromagnetic field generated by the conductor feeling the rectification caused by lightning current.

\section{MONTE CARLO RISK ASSESSMENT OF LIGHTNING DISASTERS IN RAILWAY OVERHEAD LINES}

According to the type of loss, the lightning damage risk should be divided into R1: injury or death caused by lightning; R2: unnecessary damage to public facilities and public service areas caused by lightning strikes; R3: loss of tangible cultural heritage and intangible cultural heritage; R4 : Economic loss risks to material management.

According to the different positions of the lightning strike points, the causes of lightning damage are divided into four types: S1: overhead lines damage; S2: damage near the overhead lines; S3: overhead line service equipment facilities damage; S4: damage near service equipment facilities. The damage caused by lightning strikes are divided into three types: D1: damage to personnel and other creatures in and around buildings caused by lightning strikes to buildings; D2: damage to overhead line equipment by lightning strike to overhead line; D3: overhead line facility electrical and electronic system failure caused by strong current breakdown by lightning strikes. Losses caused by lightning strikes are divided into four types: L1: damage to passengers and staff; L2: loss of public facilities and public service areas; L3: loss of tangible cultural heritage and intangible cultural heritage; L4: economic losses to material management .

Each risk component RX depends on the number of dangerous events NX, building damage probability PX and single damage rate LX:

\section{$\mathrm{RX}=\mathrm{NX} \times \mathrm{PX} \times \mathrm{LX}(\mathrm{X}=\mathrm{A}, \mathrm{B}, \mathrm{C} \ldots)$}

Finally, the calculated R1, R2, and R3 are compared with the risk tolerances specified in the standard to confirm lightning protection measures[1]. 
The methods of risk assessment can be roughly divided into two parts: qualitative and quantitative. Up to several hundred evaluation models can be used. The main methods of qualitative analysis mainly include decision makers' administrative instructions, peer review, retrospective analysis, empirical trend extrapolation, Delphi method and so on. The main methods of quantitative analysis include net present value method, GP method, analytic hierarchy process, risk value method, stress test method, KPI method, regression method, Monte Carlo method and so on.

The Monte Carlo method, also known as stochastic simulation method or statistical test method, refers to a way to solve mathematics, physics and engineering technical problems approximate solution based on probability and statistics theory, under the fact that the known distribution of distribution features is complicated, through statistical analysis of random variables, stochastic simulation.

Considering the uncertainty of building damage probability, it is impossible to take all risk factors into account during the research process. Monte Carlo simulation is an important method to statistically simulate uncertain problems and approximate the predicted results through a series of random numbers. Therefore, Monte Carlo simulation can describe the characteristics and process of building damage probability realistically, solving the problem that the traditional numerical method of building damage probability difficult to solve. The Monte Carlo simulation method is carried out by using random sampling[2]. Only when the sampling size of multiple iterations is large enough, the calculation amount is large enough to obtain an accurate calculation. The probability distribution map of Monte Carlo simulation of railway overhead line damage under different protection levels is as follows:

The overhead line damage probability is 0.8 without lightning protection. The normal distribution of overhead line damage probability without lightning protection is shown in Figure 1.

The overhead line damage probability is 0.3 under Class IV lightning protection. The normal distribution of overhead line damage probability under Class IV lightning protection is shown in Figure 2.

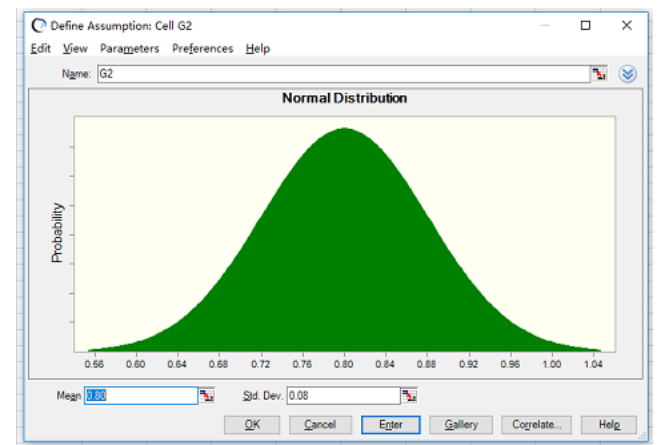

Fig. 1 The normal distribution of overhead line damage probability without lightning protection

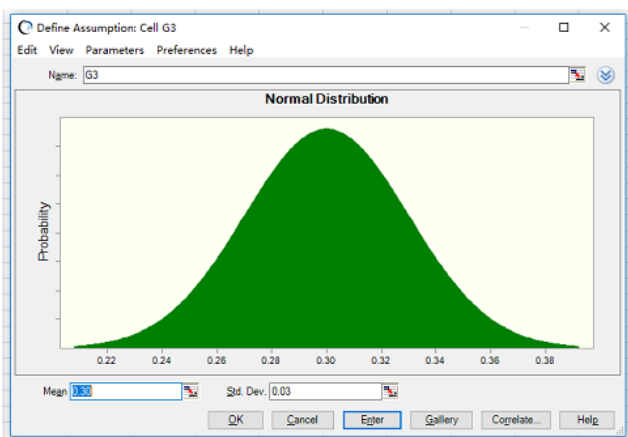

Fig. 2 The normal distribution of overhead line damage probability under Class IV lightning protection

The overhead line damage probability is 0.2 under Class III lightning protection. The normal distribution of overhead line damage probability under Class IV lightning protection is shown in Figure 3.

The overhead line damage probability is 0.1 under Class II lightning protection. The normal distribution of overhead line damage probability under Class IV lightning protection is shown in Figure 4.

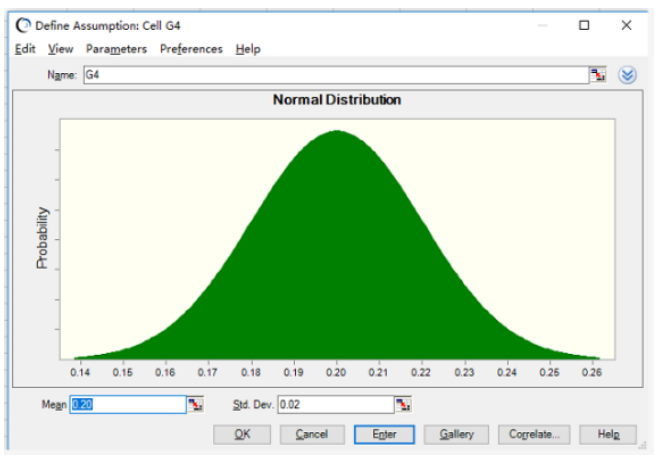

Fig. 3 The normal distribution of overhead line damage probability under Class IV lightning protection

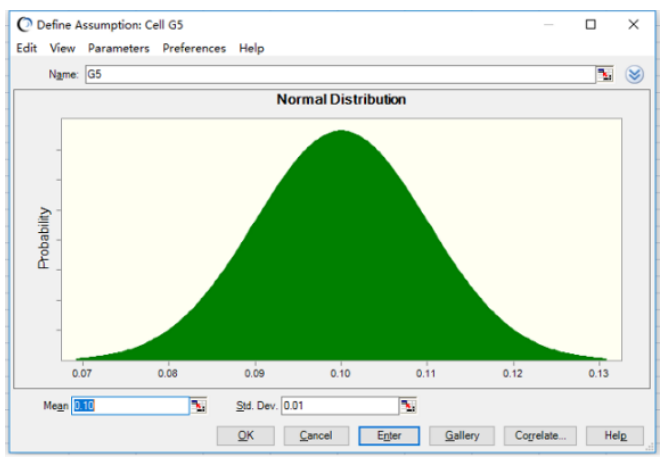

Fig. 4 The normal distribution of overhead line damage probability under Class IV lightning protection

The overhead line damage probability is 0.04 under Class I lightning protection. The normal distribution of overhead line damage probability under Class IV lightning protection is shown in Figure 5.

The overhead line damage probability is 0.03 under lightning protection installing Class I down-conductor. The 
normal distribution of overhead line damage probability under lightning protection installing Class I down-conductor is shown in Figure 6.

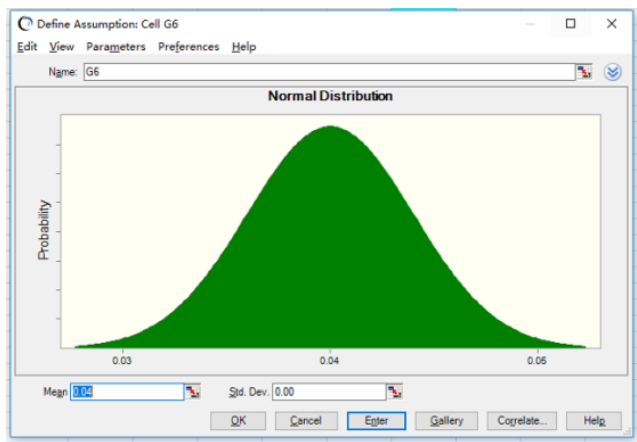

Fig. 5 The normal distribution of overhead line damage probability under Class IV lightning protection

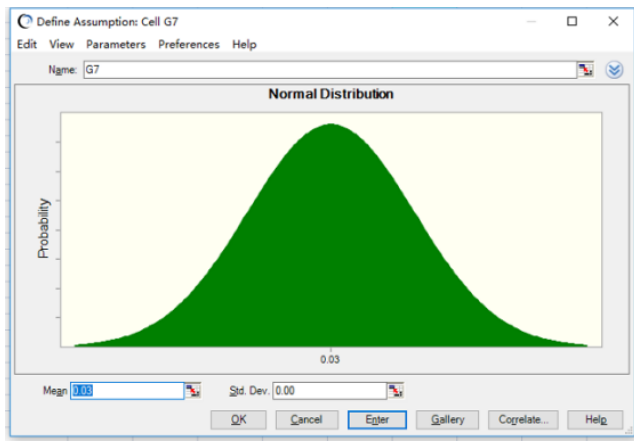

Fig. 6 The normal distribution of overhead line damage probability under lightning protection installing Class I down-conductor

The overhead line damage probability is 0.001 under lightning protection installing natural parts lightning receptor. The normal distribution of overhead line damage probability under lightning protection installing natural parts lightning receptor is shown in Figure 7.

The average frequency distribution of overhead line damage probability under different protection levels is shown in Fig. 8.

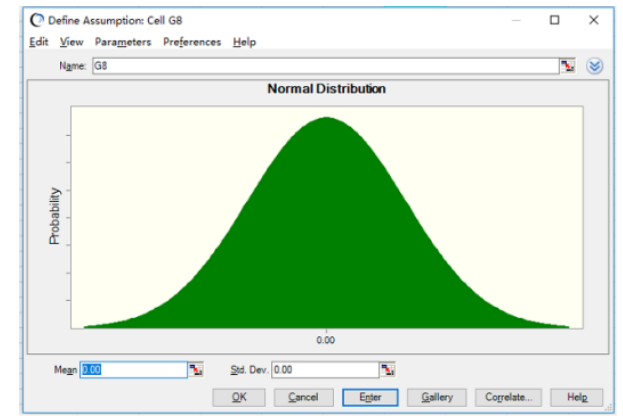

Fig. 7 The normal distribution of overhead line damage probability under lightning protection installing natural parts lightning receptor

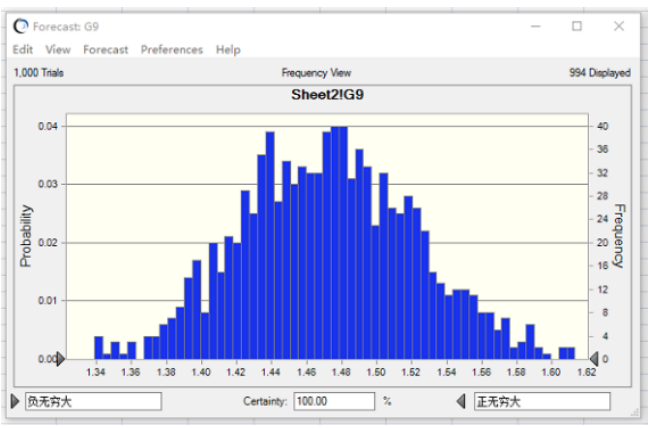

Fig. 8 The average frequency distribution of overhead line damage probability under different protection levels

The frequency distribution of overhead line damage probability under different protection levels is shown in Fig. 9.

The total frequency distribution of overhead line damage probability under different protection levels is shown in Fig. 10.

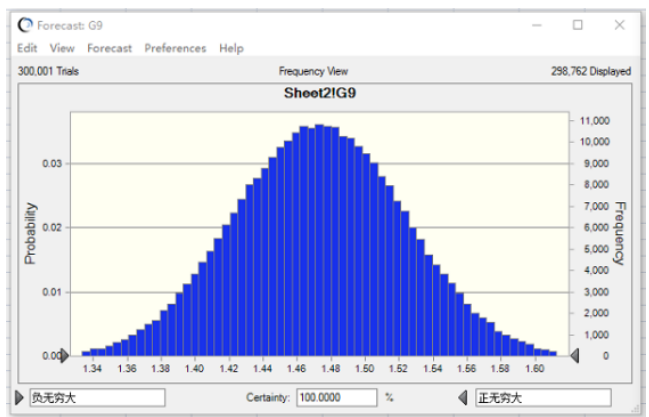

Fig. 9 The frequency distribution of overhead line damage probability under different protection levels

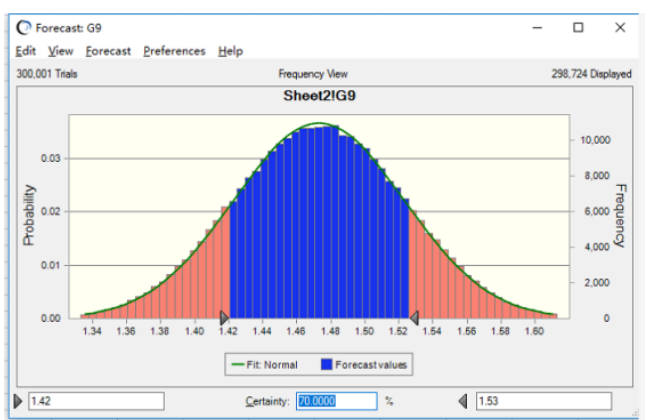

Fig. 10 The total frequency distribution of overhead line damage probability under different protection levels

\section{LIGHTNING PROTECTION CATEGORY DETERMINATION METHOD OF OVERHEAD LINE}

According to the importance, using properties, the possibility and consequences of lightning accidents of the building, It divides into three categories in GB50057-2010 on the basis of annual estimated number of strikes to building[3].

The annual estimated number of strikes to building should be calculated as follows:

$\mathrm{N}=\mathrm{k} \times \mathrm{N} \mathrm{g} \times \mathrm{A} \mathrm{e}$

Tips: $\mathrm{N}$ - the annual estimated number of lightning strikes to building (times/a); 
$\mathrm{K}$ - correction coefficient, take 1 in general; take 1.5 for building located in wet environment such as lakeside and groundwater outcrop, also take 1.5 for building located in special area such as top of mountain, mountain estuary, hillside or mountain, where soil resistivity is small ; some buildings using bricks as the main body with metal roof easily to attract lightning, if lightning-free equipment is not installed, 1.7 is taken; and buildings located on the top of the mountain or in the wilderness are easy to accumulate charge, making the air between cloud easy to broken down, so take 2 ;

$\mathrm{N}$ - - the annual average density of lightning strikes in the area where the building is located (times/km2/a);

A e-the equivalent area of the number of lightning strikes intercepted by the building.( $(\mathrm{km} 2)[4]$

Judge whether the building needs corresponding lightning protection measures and take which level of lightning protection measures according to the result.

\section{LIGHTNING ROD FOR LIGHTNING PROTECTION OF RAILWAY OVERHEAD LINE}

According to above Monte Carlo risk assessment of the railway overhead line lightning disaster and the category determination method of overhead line, in high-speed railway contact cable project example, take the method avoiding direct lightning strike, taking "lead" method, using lightning rod and lightning as lightning receptor, receiving lightning current to lead lightning current safely and quickly to earth though a good grounding device.

The high-speed railway is laid in parallel to form a cross-grid and a network of lightning protection belts is built. The intersections are welded to form a lightning protection net. The four corners should be equipped with a lightning current downconductor line to form a Faraday cage that can effectively reduce electromagnetic intrusion [5]. All parts are formed into an equal potential high-speed railway contact network lightning protection zone.

The total buried depth of grounding device is $3.0-3.5 \mathrm{~m}$, plus a 0.5-1.0 $\mathrm{t}$ composite resistance reducer, reaching a grounding resistance $\leq 1 \Omega$, effectively increasing the lightning-resistant level.

\section{CONCLUSION}

According to the characteristics of high-speed railway soil and overhead line climate, combined theory with the reality of high-speed railway overhead line, taking the lightning protection design of high-speed railway as an example, from the perspective of direct lightning damage and indirect lightning damage, analyzing and designing the lightning effect on overhead line to obtain the related research of the high-speed railway overhead line lightning and give the lightning protection measures. Compared with other overhead lines, the high-speed railway lightning over-voltage is significantly reduced and the effect make big improvements.

\section{REFERENCES}

[1] A.Ametani, T.Kawamura. A Method of a Lightning Surge Analysis Recommended in Japan using EMTP.IEEE TRANSACTIONSON POWER DELIVERY. 2005. 5(20): 867-873

[2] GRCEV L.Impulse Efficiency of Ground Electrodes[J]. IEEE Transactions on Power Delivery, 2009, 24(1): 441-451.

[3] Big data application: Study and archival of mental health data, using MongoDB. Priyanka Dhaka, Rahul Johari. Electrical, Electronics and Optimization Techniques (ICEEOT),International Conference on . 2016

[4] A secure cloud computing based framework for big data information management of smart grid. J Baek, Q H Vu,J K Liu,et al. IEEE Transactions on Cloud Computing. 2015

[5] Analysis of data storage mechanism in NoSQL database MongoDB. Gu Y,Wang X, Shen S, et al. Consumer Electronics-Taiwan (ICCE-TW), 2015 IEEE International Conference on . 2015 